\title{
Genomic reconstruction of histidine metabolism and regulation in human microbiome
}

\author{
N.V. Sernova, I.A. Zharov, G.A. Ashniev, S.A. Leyn, D.A. Rodionov* \\ Institute for Information Transmission Problems RAS, Moscow, Russia \\ *e-mail: rodionovdmitry.delmar@gmail.com
}

Key words:

Introduction: Histidine is one of the essential amino acids in mammals. Prokaryotes, fungi, and plants can synthesize it using a common biosynthesis pathway. Histidine biosynthetic pathway in Bacteria consists of ten biochemical steps. In Escherichia coli and other gamma-proteobacteria, the histidine biosynthesis genes are organized into the his operon, which is regulated via the leader peptide-dependent transcription attenuation mechanism. However, the histidine-dependent transcriptional regulation is not described in Gram-positive bacteria. The human microbiota represents a complex assemblage of microbial species and a significantly larger set of gene functions that are organized into complex metabolic and transcriptional networks. Bacteria from the human microbiome outnumber the number of human cells within a person by an order of magnitude and are represented by a large number of diverse taxa. Mapping gene functions and biological networks in the human microbiome is critically important for our understanding of mutualistic relationships including both microbe-microbe and host-microbe interactions and its relation to diseases such as obesity, diabetes, Crohn's disease.

Results: Genome-scale mapping and reconstruction of metabolic pathways and transcriptional regulatory networks in taxonomically diverse microbes is one of the critical tasks of microbial genomics. Here we present a study of distribution of histidine biosynthetic and transport genes across the reference set of 2228 bacterial genomes from 11 phyla out of sequenced consortia of Human Gut Microbiome. By using the comparative genomics approach we have reconstructed the biochemical pathways and transcriptional regulons for histidine metabolism in HMP genomes. The majority of histidine prototrophs belong to the Actinobacteria, Proteobacteria and Bacterodetes phyla that contain $90 \%, 96 \%$ and $76 \%$ of prototrophic genomes, respectively. We have performed the comparative genomics reconstruction of regulons for a novel histidine-specific transcriptional regulator HisR that is homologous to the TrpR family of tryptophan-sensing repressors. Orthologs of HisR were found in Firmicutes, Xanthomonadales and some Alphaproteobacteria. In all genomes possessing HisR orthologs, we have identified DNA binding motifs of HisR, palindromes with consensus YACTTTANYNNRNTAAAGTR, and reconstructed the respective HisR regulons that mostly include the histidine biosynthetic operons and putative histidine transporters The regulon analysis allowed us to identify novel types of transporters that are potentially involved in histidine uptake.

Conclusions: The comparative genomics-based inference of histidine metabolism, uptake and regulation networks allows improve a metabolic modelling and provide a reference dataset for interpretation of transcriptomics data for human microbiota.

Acknowledgments: This research was supported by the Russian Science Foundation (grant No. 14-14-00289). 